\title{
Photoinduced mendable network polymer from poly(butylene adipate) end-functionalized with cinnamoyl groups
}

\author{
Nobuhiro Oya, Petty Sukarsaatmadja, Kazuki Ishida and Naoko Yoshie \\ A network polymer with photoinduced mending abilities was prepared from cinnamoyl-telechelic prepolymer of poly(butylene \\ adipate), $\mathrm{PBAC}_{2}$, and a tetra-cinnamoyl linker, $\mathrm{C}_{4}$, by photodimerization. The photodimerization reaction of the cinnamoyl \\ groups was analyzed using both ultraviolet (UV)/visible (vis) and infrared (IR) spectroscopy. In the reaction, the isomerization \\ of the cinnamate group from the trans to the cis conformation competes with the dimerization reaction of cinnamates. The \\ fractions of dimer and isomer populations estimated from the UV/vis spectra revealed that dimerization was the more preferred \\ reaction at temperatures higher than the melting point of $\mathrm{PBAC}_{2}$. When a film of the network polymer was damaged by tapping, \\ the cinnamate dimers dissociated to form cinnamate monomers due to the reversibility of the dimerization. To recover the \\ network structure, these monomers could be dimerized once again through a photoirradiation process. These results show that \\ cracks in the network polymer can be recovered through photoirradiation.
}

Polymer Journal (2012) 44, 724-729; doi:10.1038/pj.2012.18; published online 14 March 2012

Keywords: cinnamate; network polymer; photodimerization; photoinduced mend

\section{INTRODUCTION}

Rising prices and the depletion of natural resources such as petroleum, combined with rising environmental concerns, have prompted the development of recyclable and reworkable polymers. Reversible polymers, which can be reverted back to their initial monomers or shorter repolymerizable oligomers by marginal stimuli, are promising for this purpose. Recently, there has been an emerging interest in exploiting the reversibility of such polymers for repairing cracks on the molecular level. ${ }^{1-3}$

Polymers degrade with age due to accumulated stress and strain, the results of which manifest themselves in the formation of microcracks. Healing polymers are capable of repairing these microcracks over the serviceable lifespan of the material. Healing properties are useful for extending the working life and enhancing the safety performance of polymeric materials. Some healing mechanisms including the incorporation of healing agents into a matrix ${ }^{4}$ and the utilization of a reversible reaction have been examined. As an example of the latter, Chen et al. ${ }^{5,6}$ synthesized a mendable polymer that was densely cross-linked using a thermally reversible Diels-Alder reaction. Cordier et al. ${ }^{7}$ reported a self-mendable elastomer networked with hydrogen bond interactions. When microcracks form in these materials, either through thermal or mechanical stress, the chemically weak dynamic bonds are preferentially cleaved. After the stress is relieved, the broken bonds reform, repairing the cracks. Mending systems based on dynamic bonding consist of chemical repair at the molecular level, which restores the original properties of the material. Moreover, these systems can even mend cracks repeatedly in the same position.

A number of publications have reported photoinduced reversible network polymers based on the dimerization of the photoreactive groups, such as benzylidenephthalimidines, ${ }^{8}$ coumarines, ${ }^{9,10}$ anthracene $^{11}$ and cinnamate. ${ }^{12,13}$ Although various applications including photolithography ${ }^{9}$ and the fabrication of liquid crystals ${ }^{14}$ have been examined, there are only a few reports on photoinduced mendable materials using photodimerization. ${ }^{15,16}$ Chung et al. ${ }^{15}$ developed a photomendable polymer using cinnamate dimer. A double network polymer was synthesized through the simultaneous processes of photoinduced radical polymerization of methacrylate monomers and photodimerization of the cinnamoyl groups in 1,1,1tris-(cinnamoyloxymethyl) ethane. Cracks in this polymer were repaired with $280 \mathrm{~nm}$ irradiation, which promoted the recovery of the 1,1,1-tris-(cinnamoyloxymethyl) ethane-based network structure. Froimwicz et al. ${ }^{16}$ presented a photomendable hyper-branched polymer based on anthracene dimers. The surface scratches of this polymer were mended in two steps. First, using irradiation at $254 \mathrm{~nm}$, anthracene dimers were de-cross-linked to liquefy the material around the scratches, and then, using $366 \mathrm{~nm}$ irradiation, the cross-links were restored removing the scratches.

In previous works, we have studied the recyclability, soft-hard conversion and mending ability of network polymers synthesized from telechelic prepolymers connected with reversible bonds. ${ }^{17-19}$ The main chain of the telechelics determines the basic performance of the 
a<smiles>CC(C)(C)OCCCCC(=O)OCCCCC(=O)OCCCCOC(=O)/C=C/c1ccccc1</smiles>

$\mathrm{PBAC}_{2}$<smiles>O=C(/C=C/c1ccccc1)OCC(COC(=O)/C=C/c1ccccc1)(COC(=O)/C=C/c1ccccc1)COC(=O)/C=C/c1ccccc1</smiles>

$\mathrm{C}_{4}$

b

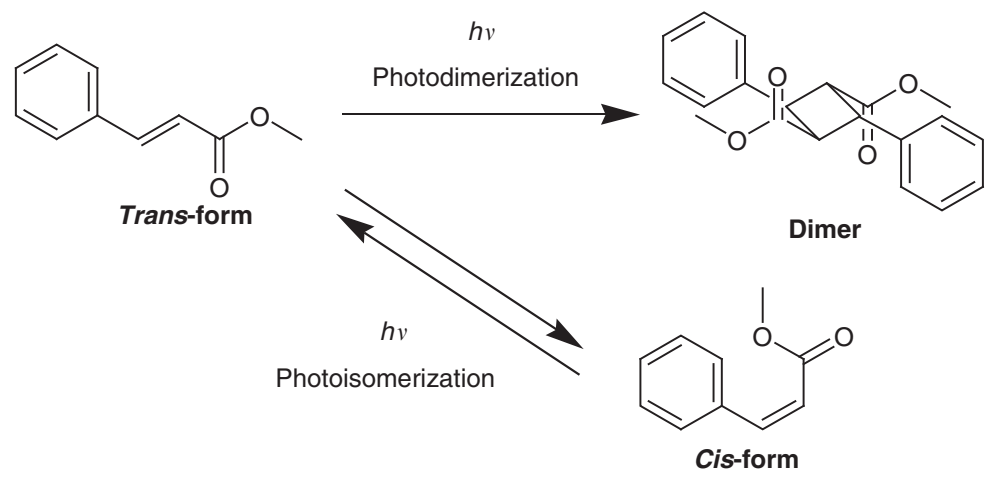

Figure 1 Molecular structures of $\mathrm{PBAC}_{2}$ and $\mathrm{C}_{4}(\mathbf{a})$ and photoreactions of cinnamate (b).

polymers, while their functionality is regulated by their reversible groups. Therefore, the functionalities and the basic performance can be designed independently in these network polymers. In this study, we synthesized a photoinduced mendable network polymer from a cinnamoyl-telechelic poly(butylene adipate) $\left(\mathrm{PBAC}_{2}\right)$ and a tetracinnamoyl linker $\left(\mathrm{C}_{4}\right)$ connected via the photodimerization of the cinnamoyl groups (Figure 1). The photopolymerization and photomending behaviors were analyzed with UV/vis and attenuated total reflection-IR spectroscopies.

\section{EXPERIMENTAL PROCEDURE}

\section{Materials}

Poly(butylene adipate) diol (PBAdiol, $M_{\mathrm{n}}^{\mathrm{NMR}}=1000$. Gel permeation chromatography (GPC): $M_{\mathrm{n}}{ }^{\mathrm{GPC}}=1010 ; M_{\mathrm{w}} / M_{\mathrm{n}}=3.28$ ) was obtained from Aldrich Chemical Co. (St Louis, MO, USA) Pentaerythritol and cinnamoyl chloride were purchased from Tokyo Chemical Industry Co. Ltd (Tokyo, Japan). Chloroform and methanol were purchased from Wako Pure Chemical Industries, Ltd (Osaka, Japan). Ethanol was purchased from Nacalai Tesque, Inc. (Kyoto, Japan). Anhydrous tetrahydrofuran was purchased from Kanto Chemical Co. (Tokyo, Japan). All reagents and solvents were used as received.

\section{Analytical procedures}

Ultraviolet (UV)/visible (vis) absorption spectra were collected on a U-3010 Hitachi spectrophotometer (Hitachi High-Tech Co., Tokyo, Japan). GPC measurements were performed on a Tosoh HLC 8220 GPC (Tosoh Co., Tokyo, Japan) system equipped with two TSK-Gel $\mathrm{GMH}_{\mathrm{HR}^{-}} \mathrm{N}$ columns purchased from Tosoh Co. (chloroform, $40^{\circ} \mathrm{C}, 1 \mathrm{mg} \mathrm{ml}^{-1}$ ). Polystyrene standards with low polydispersity were used to construct a calibration curve. ${ }^{1} \mathrm{H}-\mathrm{NMR}$ (nuclear magnetic resonance) spectra of chloroform- $d$ solutions were measured using a JEOL JNM-ECS400 NMR SYSTEM (JEOL Ltd., Tokyo,
Japan) at room temperature. Fast atom bombardment mass spectroscopy measurements were performed using a JEOL JMS-600H. Attenuated total reflection-infrared spectroscopy was measured with a Thermo Scientific Nicolet iS10 equipped with a Smart iTR (Thermo Fisher Scientific Inc., Waltham, MA, USA) accessory and a ZnSe crystal. Differential scanning calorimetry was performed using a Perkin-Elmer Pyris 1 (Perkin-Elmer Inc, Waltham, MA, USA) under a $\mathrm{N}_{2}$ atmosphere. Thermograms were recorded for samples in aluminum pans. The melting point $\left(T_{\mathrm{m}}\right)$ and heat of fusion $\left(\Delta H_{\mathrm{f}}\right)$ were taken as the top of the peak and area under the melting endotherm, respectively, for a heating rate of $10^{\circ} \mathrm{C} \mathrm{min}-1$ after crystallization at $20^{\circ} \mathrm{C}$ for $4 \mathrm{~min}$. Heat flow during isothermal crystallization was also observed at $28^{\circ} \mathrm{C}$. All samples were thermally treated by initially heating them to $60^{\circ} \mathrm{C}$ followed by quenching to their crystallization temperature.

\section{Synthesis of $\mathrm{PBAC}_{2}$}

PBAdiol (2.81 g) and cinnamoyl chloride (7.07g, 15 e.q.) were dissolved in dehydrated tetrahydrofuran $(40 \mathrm{ml})$ under a $\mathrm{N}_{2}$ purge and then stirred under reflux for 3 days. After cooling to room temperature, the product was precipitated using excess ethanol at $0^{\circ} \mathrm{C}$ and then dried under vacuum. Cinnamoyl-terminated PBA was obtained after dissolution in chloroform, followed with precipitation using methanol at $0{ }^{\circ} \mathrm{C}$ and subsequent drying under vacuum. The yield was $1.074 \mathrm{~g} .{ }^{1} \mathrm{H}-\mathrm{NMR}(400 \mathrm{MHz}$, chloroform- $d$ ): $\delta 7.71(\mathrm{~d}, 2 \mathrm{H}), 7.55-7.38(\mathrm{~m}, 10 \mathrm{H}), 6.46(\mathrm{~d}, 2 \mathrm{H}), 4.09-4.24(\mathrm{~m}, 55 \mathrm{H}), 2.33$ $(\mathrm{m}, 51 \mathrm{H}), 1.64-1.79\left(\mathrm{~m}\right.$, overlapped with $\mathrm{H}_{2} \mathrm{O}$ peak $), M_{\mathrm{n}}^{\mathrm{NMR}}=2900$, from the relative areas of the cinnamate peak at 6.46 p.p.m. and the PBA peak at 2.33 p.p.m. GPC: $M_{\mathrm{n}}{ }^{\mathrm{GPC}}=4500 ; M_{\mathrm{w}} / M_{\mathrm{n}}=1.27$.

\section{Synthesis of $\mathrm{C}_{4}$}

A mixture of pentaerythritol $(0.163 \mathrm{~g}, 1.2 \mathrm{mmol})$ and cinnamoyl chloride $(1.00 \mathrm{~g}, 6 \mathrm{mmol})$ was maintained at $120^{\circ} \mathrm{C}$ for $3 \mathrm{~h}$. After cooling, the crude product was dissolved in hot ethanol and then recrystallized from the solution. 
After filtration, a product was obtained with a $49 \%$ yield $(1.92 \mathrm{~g}) .{ }^{1} \mathrm{H}-\mathrm{NMR}$ $(400 \mathrm{MHz}$, chloroform- $d)$ : $\delta 7.71(\mathrm{~d}, 1 \mathrm{H}), 7.49(\mathrm{~m}, 2 \mathrm{H}), 7.35(\mathrm{~m}, 3 \mathrm{H}), 6.46$ $(\mathrm{d}, 1 \mathrm{H}), 4.47(\mathrm{~s}, 2 \mathrm{H})$; fast atom bombardment mass spectroscopy: 657.4 $(\mathrm{MH}), \mathrm{C}_{41} \mathrm{H}_{36} \mathrm{O}_{8}$, calculated 656.24. Differential scanning calorimetry: $T_{\mathrm{m}}=126^{\circ} \mathrm{C}$.

\section{Photopolymerization of $\mathrm{PBAC}_{2}$ and $\mathrm{C}_{4}$}

Thin films of $\mathrm{PBAC}_{2}$ and $\mathrm{C}_{4}$ with a 1:1 molar ratio were prepared on quartz plates by spin-coating from a dilute chloroform solution at 2000 r.p.m. These films were photoirradiated with a wavelength band of $300-400 \mathrm{~nm}$ using a $100 \mathrm{~W}$ xenon lamp (LAX-Cute, Asahi spectra Co. Ltd, Tokyo, Japan) with a UVA mirror module.

\section{Mendability test}

Spin-coated film (thickness $\approx 0.01 \mathrm{~mm}$ ) of $1: 1$ molar $\mathrm{PBAC}_{2}: \mathrm{C}_{4}$ on quartz was polymerized with $300-400 \mathrm{~nm}$ photoirradiation for $10 \mathrm{~min}$ at $60^{\circ} \mathrm{C}$. The film was then covered with a thin glass plate (Matsunami Glass Ind., Ltd, Osaka, Japan, micro cover glass no. 1) and repeatedly tapped gently. Light with a $300-400 \mathrm{~nm}$ wavelength was irradiated onto the tapped samples for $10 \mathrm{~min}$ at $60^{\circ} \mathrm{C}$. After each step, UV/vis absorption spectrums were collected.

\section{RESULTS AND DISCUSSION}

Photoreaction of $\mathrm{PBAC}_{2}$ with $\mathrm{C}_{4}$

$A \mathrm{PBAC}_{2}$ polymer with a degree of polymerization of 13 was prepared by the esterification of PBAdiol with cinnamoyl chloride. In the ${ }^{1} \mathrm{H}$ NMR spectra of $\mathrm{PBAC}_{2}$, no peaks ascribed to the chain ends of the PBAdiol were observed, indicating the successive synthesis of $\mathrm{PBAC}_{2}$.

The photoreactivity of the cinnamoyl groups on $\mathrm{PBAC}_{2}$ and $\mathrm{C}_{4}$ in their bulk states were monitored by UV/vis absorption spectroscopy. Figure $2 \mathrm{a}$ shows the temporal evolution of the UV/vis absorption spectra for the 1:1 molar mixture of $\mathrm{PBAC}_{2}$ and $\mathrm{C}_{4}$ during photoirradiation $(\lambda=300-400 \mathrm{~nm})$ at $60^{\circ} \mathrm{C}$. The absorption at $280 \mathrm{~nm}$ coming from the cinnamoyl group reduceed with irradiation time, indicating that the dimerization of the cinnamoyl groups was promoted by photoirradiation.

Attenuated total reflection-infrared spectroscopy also showed the formation of the cinnamoyl dimers (Figure 3). In the spectra of the 1:1 mixture before photoirradiation, the $\mathrm{C}=\mathrm{C}$ and $\mathrm{C}=\mathrm{O}$ stretching bands of the cinnamoyl group appeared at $1636 \mathrm{~cm}^{-1}$ and $1716 \mathrm{~cm}^{-1}$ (as a shoulder), respectively. ${ }^{20}$ After irradiation with $300-400 \mathrm{~nm}$ light for $10 \mathrm{~min}$, these characteristic peaks had disappeared, indicating consumption of the cinnamoyl groups.

It is well known that cinnamate takes on the trans-conformation under ambient conditions. After irradiation with UV light, both the dimerization via $(2+2)$ cycloaddition and isomerization cause the cinnamate to take on the cis-form (Figure 1). Although the dimer has no absorption peaks between 250 and $350 \mathrm{~nm}$, both the trans- and cis-monomers have absorption peaks at $\approx 280 \mathrm{~nm}$. If the trans-cis isomerization solely proceeds, an isosbestic point appears at $250 \mathrm{~nm} \cdot{ }^{21}$ The disappearance of the isosbestic point in the spectra clearly showed that dimerization competes with isomerization in the sample during photoirradiation (Figure 2a).

The UV/vis absorption spectra can be analyzed quantitatively based on the following equations: ${ }^{22}$

$$
\begin{aligned}
& f_{T}=\frac{\varepsilon_{T}}{\varepsilon_{T}-\varepsilon_{C}}\left(\frac{D_{\max }^{t}}{D_{\text {max }}^{0}}-\frac{\varepsilon_{C} D_{\text {iso }}^{t}}{\varepsilon_{T} D_{\text {iso }}^{0}}\right) \\
& f_{C}=\frac{\varepsilon_{T}}{\varepsilon_{T}-\varepsilon_{C}}\left(\frac{D_{\text {iso }}^{t}}{D_{\text {iso }}^{0}}-\frac{D_{\text {max }}^{t}}{D_{\text {max }}^{0}}\right) \\
& f_{D}=\left(1-\frac{D_{\text {iso }}^{t}}{D_{\text {iso }}^{0}}\right)
\end{aligned}
$$
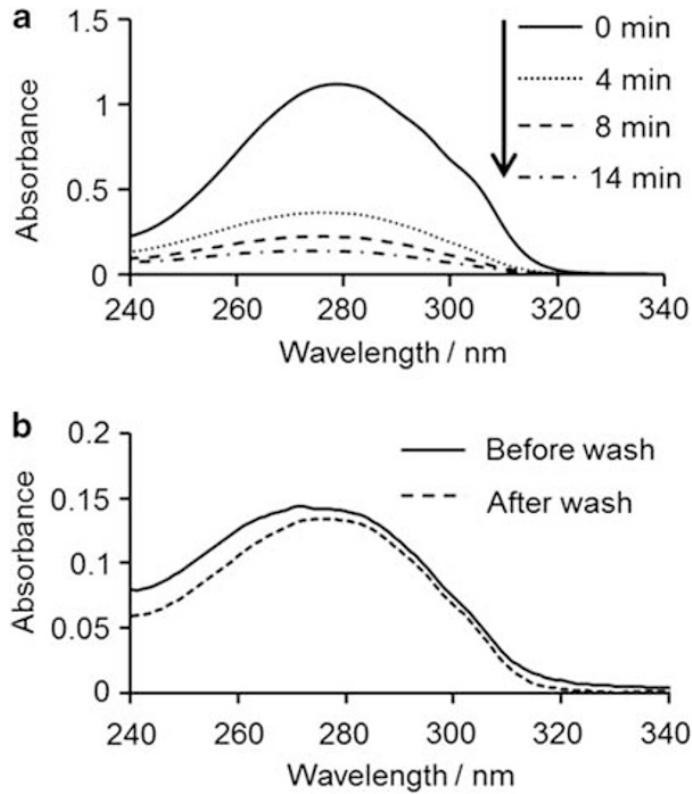

Figure $2 \mathrm{UV}$-vis spectra of (a) 1:1 molar $\mathrm{PBAC}_{2}: \mathrm{C}_{4}$ film on quartz during photoirradiation $(\lambda=300-400 \mathrm{~nm})$ at $60^{\circ} \mathrm{C}$ for $0,4,8$ and $14 \mathrm{~min}$ and $(\mathrm{b})$ photodimerized 1:1 molar $\mathrm{PBAC}_{2}: \mathrm{C}_{4}$ film before (solid line) and after (dashed line) chloroform wash.

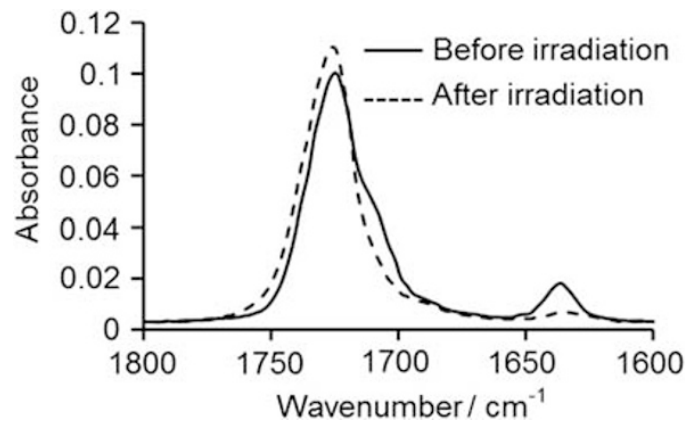

Figure 3 Changes in the attenuated total reflection-infrared spectra of 1:1 molar $\mathrm{PBAC}_{2}: \mathrm{C}_{4}$ by photoirradiation at $60^{\circ} \mathrm{C}$; before irradiation (solid line) and after irradiation for $10 \mathrm{~min}$ (broken line).

where subscripts $T, C$ and $D$ denote the trans-cinnamate, ciscinnamate and dimer, respectively; $f$ is the mole fraction; $\varepsilon$ is the molar extinction coefficients at $271 \mathrm{~nm}$ and $D_{\mathrm{sso}}^{t}$ and $D_{\max }^{t}$ are, respectively, the optical densities at $250 \mathrm{~nm}$ (isobestic point of isomerization) and $271 \mathrm{~nm}$ at time $t$. Using these equations and the extinction coefficient data from Rennert et al., ${ }^{23}$ the progress of the dimerization and isomerization in the $1: 1$ molar $\mathrm{PBAC}_{2}: \mathrm{C}_{4}$ mixture at $60{ }^{\circ} \mathrm{C}$ was estimated as shown in Figure 4. Initially, the isomerization to the cis-form proceeded quickly, followed by the gradual progress of the dimerization reaction. The photoreaction reached a stationary state after $14 \mathrm{~min}$. At this point, the dimer had became the primary product.

The photopolymerization reactions of pure $\mathrm{PBAC}_{2}$ at room temperature were also analyzed. The irradiation time required to reach a photostationary state as well as the fractional populations of 


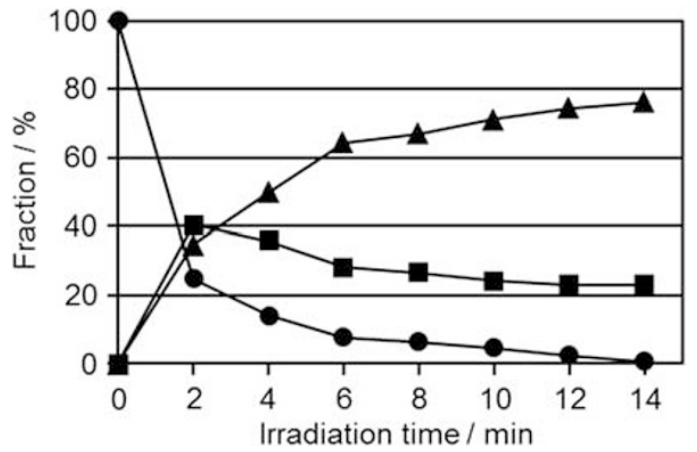

Figure 4 Simultaneous progress of cycloaddition and trans-cis isomerization in 1:1 molar $\mathrm{PBAC}_{2}: \mathrm{C}_{4}$ mixture during photoirradiation; fractional populations of trans-cinnamate (circle), cis-cinnamate (square) and dimer (triangle) are plotted as a function of irradiation time.

Table 1 Fractional populations of trans-and cis-isomers and dimer of the cinnamoly groups in the photostationary state

\begin{tabular}{lcccccc}
\hline & & \multicolumn{3}{c}{ Time to reach } \\
stationary \\
& & & & & & \\
& & Photoreaction & state & Trans & Cis & Dimer \\
Entry & Sample & temperature & $(s)$ & $(\%)$ & $(\%)$ & $(\%)$ \\
\hline 1 & $\mathrm{PBAC}_{2}$ & Room temperature & 540 & 13 & 55 & 32 \\
2 & $\mathrm{PBAC}_{2}$ & $60^{\circ} \mathrm{C}$ & 2100 & 1 & 31 & 68 \\
3 & $1 / 1 \mathrm{PBAC}_{2} / \mathrm{C}_{4}{ }^{a}$ & Room temperature & 420 & 41 & 16 & 43 \\
4 & $1 / 1 \mathrm{PBAC}_{2} / \mathrm{C}_{4}{ }^{\mathrm{a}}$ & $60^{\circ} \mathrm{C}$ & 840 & 1 & 23 & 76 \\
\hline
\end{tabular}

aMixture of $\mathrm{PBAC}_{2}$ and $\mathrm{C}_{4}$ in 1:1 molar ratio.

the dimer and two isomeric monomers in that state are summarized in Table 1. The cinnamate dimer was the main component of the photoreactions at $60^{\circ} \mathrm{C}$ (entry 2,4), while more trans- and cis-monomers remained in the stationary state at room temperature (entry 1,3). This temperature dependence can be explained by the difference in the molecular mobilities of the compounds. The melting point of $\mathrm{PBAC}_{2}$ is $44^{\circ} \mathrm{C}$. At temperatures below this melting point, the mobilities of the cinnamoyl groups are restricted, promoting the progress of isomerization. More trans-monomers exist in the 1:1 molar $\mathrm{PBAC}_{2}: \mathrm{C}_{4}$ mixture (entry 3 ) than in $\mathrm{PBAC}_{2}$ (entry 1 ). Because four cinnamoyl groups are packed into $\mathrm{C}_{4}$, the cis-isomers may be less sterically stable at room temperature. In the molten state $\left(60^{\circ} \mathrm{C}\right)$, higher molecular mobility assists in the dimerization. At each temperature, the 1:1 mixture reached the stationary state faster than pure $\mathrm{PBAC}_{2}$. The stationary state of the $1: 1 \mathrm{PBAC}_{2}: \mathrm{C}_{4}$ contained more dimer than that of pure $\mathrm{PBAC}_{2}$. These results can be explained by the difference in the concentration of the cinnamoyl groups, which is calculated from the molecular weight of $\mathrm{PBAC}_{2}\left(M_{\mathrm{n}}{ }^{\mathrm{NMR}}=2900\right)$ and $\mathrm{C}_{4}$ (molecular weight $\left.=656\right)$. Only $1 \mathrm{~mol}$ of the cinnamoyl groups are contained in $1500 \mathrm{~g}$ of pure $\mathrm{PBAC}_{2}$, whereas $600 \mathrm{~g}$ of the polymer mixture contains $1 \mathrm{~mol}$ of the cinnamoyl groups. This higher concentration resulted in more encounters between the two monomers, thus promoting dimerization.

The formation of network structure in the photopolymerized samples was examined using solubility testing. When the photoreacted film of pure $\mathrm{PBAC}_{2}$ was immersed in chloroform, the sample
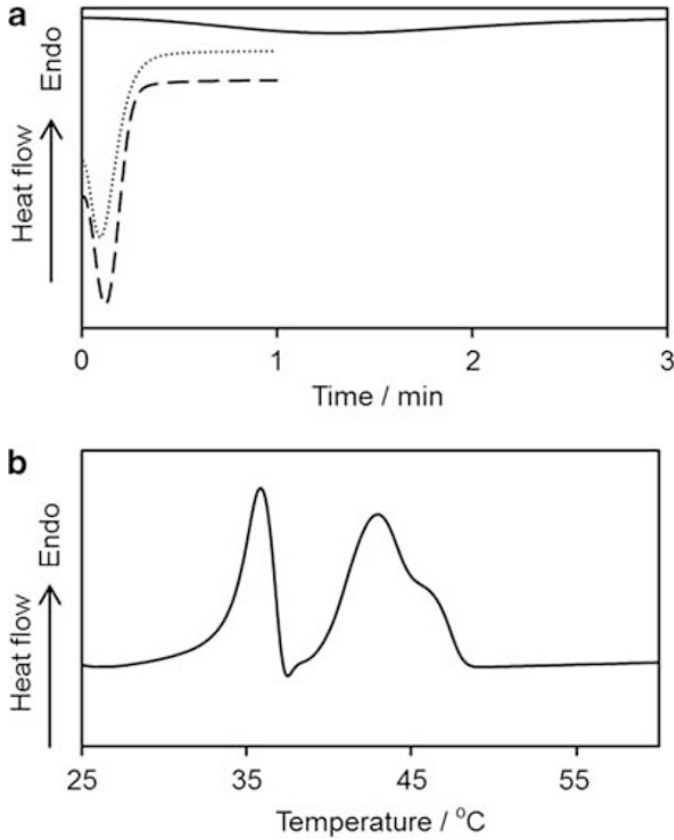

Figure 5 (a) Differential scanning calorimetry (DSC) curves during isothermal crystallization at $28^{\circ} \mathrm{C}$ of pure $\mathrm{PBAC}_{2}$ before (broken line) and after (dotted line) photopolymerization and a 1:1 molar mixture of $\mathrm{PBAC}_{2} / \mathrm{C}_{4}$ after photopolymerization (solid line). (b) DSC curves of the photopolymerized $1: 1$ molar $\mathrm{PBAC}_{2}: \mathrm{C}_{4}$ mixture with a heating rate of $10^{\circ} \mathrm{Cmin}^{-1}$.

was completely washed away. Only the linear polymer of PBA was synthesized by this photopolymerization. In contrast, the photoreacted film of the 1:1 molar $\mathrm{PBAC}_{2}: \mathrm{C}_{4}$ polymer mixture experienced little solvation in chloroform. After immersion of this film in chloroform, the intensity of the UV/vis absorption did not change compared with the absorption before immersion (Figure $2 b$ ). An insoluble network structure was formed by the dimerization of the cinnamoyl groups in this mixture. The photodimerization in the mixture polymer linked the $\mathrm{PBAC}_{2}$ and $\mathrm{C}_{4}$ together, forming the network structure. Cinnamoyl dimers, however, must also be formed between $\mathrm{C}_{4}$ linkers and between $\mathrm{PBAC}_{2}$ prepolymers.

Isothermal crystallization of pure $\mathrm{PBAC}_{2}$ before and after photoirradiation as well as isothermal crystallization of the polymer mixture after photoirradiation was observed at $28^{\circ} \mathrm{C}$ after quenching from the molten state (Figure 5a). The crystallization of $\mathrm{PBAC}_{2}$ both before and after photoirradiation was complete within $30 \mathrm{~s}$, while that of the 1:1 $\mathrm{PBAC}_{2}: \mathrm{C}_{4}$ mixture after photoirradiation took $3 \mathrm{~min}$. This slow crystallization indicates that photodimerization occurs between the cinnamoyl groups of $\mathrm{PBAC}_{2}$ and $\mathrm{C}_{4}$ to form network structures. These cross-links make the crystallization of PBA slower.

Figure $5 \mathrm{~b}$ shows the differential scanning calorimetry heating curves of photopolymerized 1:1 $\mathrm{PBAC}_{2}: \mathrm{C}_{4}$ crystallized from melting at $20{ }^{\circ} \mathrm{C}$ for $4 \mathrm{~min}$. The curve has three endothermic peaks. The heating scans with various heating rates showed that the highest temperature peak can be ascribed to the melt-recrystallization during the heating scan. The middle temperature peak at $43{ }^{\circ} \mathrm{C}$ corresponds to the melting point of PBA $\left(T_{\mathrm{m}} \approx 48^{\circ} \mathrm{C}\right)$. Photodimerization between the $\mathrm{PBAC}_{2}$ yielded long linear chains, which formed crystals similar to pure PBA. The lowest temperature peak $\left(36^{\circ} \mathrm{C}\right)$ can be ascribed to the melting of PBA near the cross-links made by the photoreaction of $\mathrm{PBAC}_{2}$ and $\mathrm{C}_{4}$. 


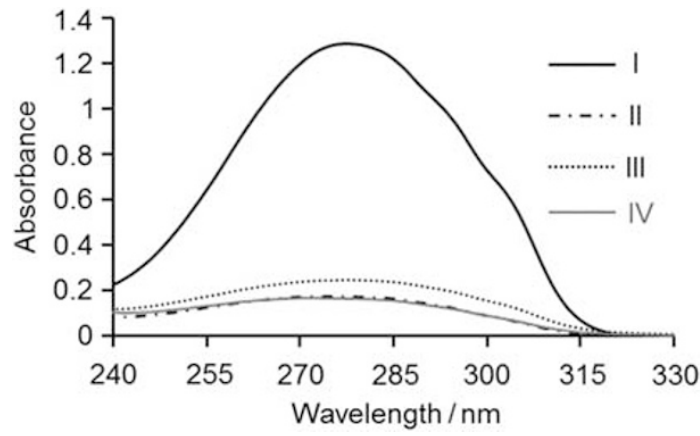

Figure $6 \mathrm{UV} / \mathrm{vis}$ spectra of $1: 1$ molar $\mathrm{PBAC}_{2}: \mathrm{C}_{4}$ polymer (I) as-cast, (II) aspolymerized, (III) as-damaged and (IV) after mending by re-photoirradiation.

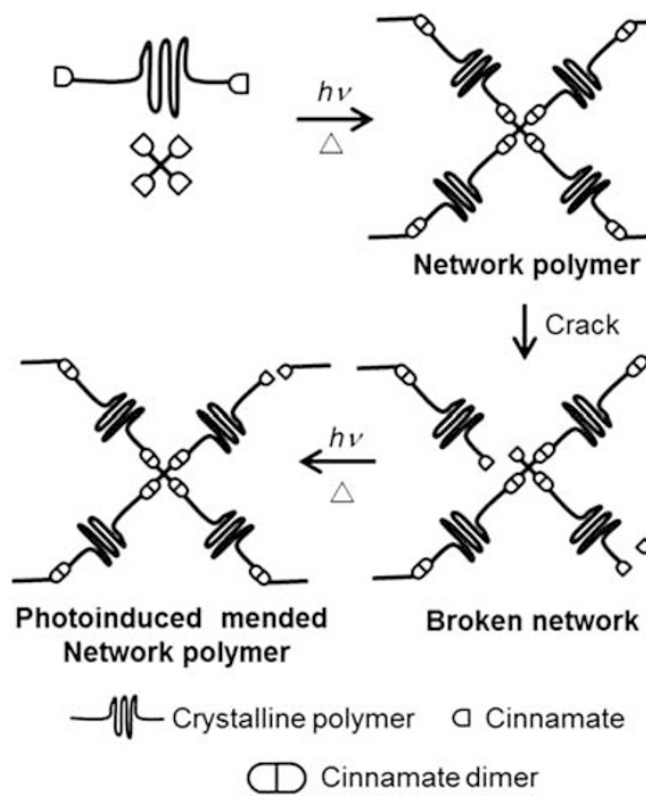

Figure 7 Schematic representation of the mending mechanism in $\mathrm{PBAC}_{2} / \mathrm{C}_{4}$.

\section{Mendability of photopolymerized 1:1 $\mathrm{PBAC}_{2}: \mathrm{C}_{4}$}

The mending ability of the polymer mixture was analyzed with the comparison of the UV/vis spectra of the samples as-cast, aspolymerized, as-damaged and as-mended by photoirradiation. The spectra are shown in Figures 6 and 7 show a schematic representation of the mending mechanism for $\mathrm{PBAC}_{2}: \mathrm{C}_{4}$ network polymer mixtures. In the UV/vis absorption spectrum of a thin film of $1: 1 \mathrm{PBAC}_{2}: \mathrm{C}_{4}(\mathrm{I})$, the characteristic peak of cinnamate was observed at $280 \mathrm{~nm}$. When the film was photoirradiated with $300-400 \mathrm{~nm}$ light for $10 \mathrm{~min}$ at $60{ }^{\circ} \mathrm{C}$, the cinnamate absorbance was significantly decreased (II), indicating the progress of the photodimerization reaction. This photodimerized polymer film was then covered with a glass plate and damaged by tapping it with a finger many times. The cinnamate absorbance at $280 \mathrm{~nm}$ slightly increased again (III), indicating the partial cleavage of cyclobutane structure in the dimer converting to the original cinnamate structure. Because of its large ring strain, the cyclobutane structure is fragile enough to be broken by applying mechanical stress. After re-photoirradiation to the damaged film, the
Table 2 Change in the fractional populations of isomers and dimer of the cinnamoly groups during mending experiment

\begin{tabular}{lrrr}
\hline Step & $\begin{array}{l}\text { Trans } \\
(\%)\end{array}$ & $\begin{array}{l}\text { Cis } \\
(\%)\end{array}$ & $\begin{array}{l}\text { Dimer } \\
(\%)\end{array}$ \\
\hline I & 100 & 0 & 0 \\
II & 3 & 20 & 77 \\
III & 3 & 30 & 67 \\
IV & 0 & 26 & 74 \\
\hline
\end{tabular}

absorbance at $280 \mathrm{~nm}$ reverted back to the original intensity before tapping (IV), indicating that the cinnamate groups in the damaged films were dimerized to once again form cyclobutane rings. These results demonstrate the photoreversible mending property of the $\mathrm{PBAC}_{2}: \mathrm{C}_{4}$ system.

The progress of dimerization during the mending process was calculated based on equations 1-3. The fractional populations of the dimer and two isomeric monomers at each stage are summarized in Table 2. After the initial photoirradiation, the amount of the transmonomer reduced to $3 \%$, and the amounts of the dimer and the cismonomer grew to $77 \%$ and $20 \%$, respectively (II). The mechanical damage inflicted on the sample resulted in the cleavage of the cyclobutane rings used to generate cis-cinnamate (III). In all, 10\% of the dimer was monomerized back to the cis-forms. By reirradiating the sample, the dimerization proceeded again (IV). The amount of dimer recovered to $74 \%$. This shows that the cyclobutane rings broken by mechanical stress can be repaired by photoirradiation in the $\mathrm{PBAC}_{2}: \mathrm{C}_{4}$ system. The recovery of the broken covalent bonds by photoirradiation can repress macrocrack formation. In addition, during this damaging and mending process, dimers located away from the damaged sections remain intact. This robust structure allows for maintenance of the sample shape.

\section{CONCLUSIONS}

A telechelic prepolymer having cinnamate end groups, $\mathrm{PBAC}_{2}$, and pentaerythritol tetracinnamate, $\mathrm{C}_{4}$, were synthesized and cross-linked by the photodimerization of the cinnamoyl groups to obtain a network polymer. The progression of the cross-linking reaction was analyzed using UV/vis and infrared spectroscopy. Although the transcis isomerization of the cinnamoyl groups competes with dimerization, more dimerization was found to occur at temperatures higher than the melting point of $\mathrm{PBAC}_{2}$. The mending behavior of the network polymer was also analyzed by UV/vis spectroscopy. When the cross-linked polymer was damaged, the cinnamate dimers along the crack surfaces dissociated into cinnamate monomers. Photoirradiation of the damaged sample led to the re-dimerization of the cinnamate monomers and the mending of the network polymer along the crack surfaces. The mending process results in the complete recovery of the chemical structure of the polymer. Although the prepolymers used in this study were telechelics of PBA, various photomendable polymers can be obtained by simply replacing the main chain of the telechelics.

\section{ACKNOWLEDGEMENTS}

This work was supported in part by the Global COE Program for Chemistry Innovation through Cooperation of Science and Engineering from the Ministry of Education, Culture, Sports, Science and Technology, Japan. 
1 van der Zwaag, V. (ed) Self Healing Materials: An Alternative Approach to 20 Centuries of Materials Science 1-385 (Springer, Netherlands, 2007).

2 Mauldin, T. C. \& Kessler, M. R. Self-healing polymers and composites. Int. Mater. Rev. 55, 317-346 (2010).

3 Wu, D. Y., Meure, S. \& Solomon, D. Self-healing polymeric materials: A review of recent developments. Prog. Polym. Sci. 33, 479-522 (2008).

4 White, S. R., Sottos, N. R., Geubelle, P. H., Moore, J. S., Kessler, M. R., Sriram, S. R., Brown, E. N. \& Viswanathan, S. Autonomic healing of polymer composites. Nature 409, 794-797 (2001).

5 Chen, X. X., Dam, M. A., Ono, K., Mal, A., Shen, H. B., Nutt, S. R., Sheran, K. \& Wudl, F. A thermally re-mendable cross-linked polymeric material. Science 295, 1698-1702 (2002).

6 Chen, X. X., Wudl, F., Mal, A. K., Shen, H. B. \& Nutt, S. R. New thermally remendable highly cross-linked polymeric materials. Macromolecules 36, 1802-1807 (2003).

7 Cordier, P., Tournilhac, F., Soulie-Ziakovic, C. \& Leibler, L. Self-healing and thermoreversible rubber from supramolecular assembly. Nature 451, 977-980 (2008).

8 Suh, D. H., Hayashi, Y. \& Ichimura, K. Polymethacrylates with benzylidenephthalimidine side chains, 1 . Photochemical characteristics of model compounds and polymers. Macromol. Chem. Phys. 199, 363-373 (1998).

9 Obi, M., Morino, S. \& Ichimura, K. The reversion of photoalignment direction of a liquid crystal induced by a polymethacrylate with coumarin side chains. Macromol. Rapid Commun 19, 643-646 (1998).

10 Jackson, P. O \& O'Neil, M. An investigation of the role of cross-linking and photodegradation of side-Chain coumarin polymers in the photoalignment of liquid crystals. Chem. Mater. 13, 694-703 (2001).

$11 \mathrm{Li}, \mathrm{T}$., Chen, J., Mitsuishi, M. \& Miyashita, T. Photolithographic properties of ultrathin polymer Langmuir-Blodgett films containing anthracene moieties. J. Mater. Chem. 13 1565-1569 (2003).

12 Kihara, H. \& Tamaoki, N. In situ photochemical conversion from cinnamoyl-functionalized liquid-crystalline monomers to liquid-crystalline dimers. Liquid Crystals 34, 1337-1347 (2007).
13 Furumi, S. \& Ichimura, K. Surface-assisted photoalignment of discotic liquid crystals by nonpolarized light irradiation of photo-cross-linkable polymer thin films. J. Phys. Chem. B 111, 1277-1287 (2007).

14 Kawatsuki, N., Goto, K., Kawakami, T. \& Yamamoto, T. Reversion of alignment direction in the thermally enhanced photoorientation of photo-cross-linkable polymer liquid crystal films. Macromolecules 35, 706-713 (2002).

15 Chung, C. M., Roh, Y. S., Cho, S. Y. \& Kim, J. G. Crack healing in polymeric materials via photochemical $[2+2]$ cycloaddition. Chem. Mater. 16, 3982-3983 (2004).

16 Froimowicz, P., Frey, H. \& Landfester, K. Towards the generation of self-healing materials by means of a reversible photo-induced approach. Macromol. Rapid Commun. 32, 468-473 (2011).

17 Watanabe, M. \& Yoshie, N. Synthesis and properties of readily recyclable polymers from bisfuranic terminated poly(ethylene adipate) and multi-maleimide linkers. Polymer 47, 4946-4952 (2006).

18 Ishida, K. \& Yoshie, N. Two-way conversion between hard and soft properties of semicrystalline cross-linked polymer. Macromolecules 41, 4753-4757 (2008).

19 Yoshie, N., Watanabe, M., Araki, H. \& Ishida, K. Thermo-responsive mending of polymers crosslinked by thermally reversible covalent bond: Polymers from bisfuranic terminated poly(ethylene adipate) and tris-maleimide. Polym. Degrad. Stab. 95, 826-829 (2010).

20 Ali, A. H. \& Srinivasan, K. S. V. Photoresponsive functionalized vinyl cinnamate polymers: synthesis and characterization. Polym. Int. 43, 310-316 (1997).

21 Nakayama, Y. \& Matsuda, T. Photocycloaddition-induced preparation of nanostructured, cyclic polymers using biscinnamated or biscoumarinated oligo(ethylene glycol)s. J. Polym Sci.: Part A: Polym. Chem. 43, 3324-3336 (2005).

22 Egerton, L., Pitts, E. \& Reiser, A. Photocycloaddition in solid poly(vinyl cinnamate). The photoreactive polymer matrix as an ensemble of chromophore sites. Macromolecules 14, 95-100 (1981).

23 Rennert, J., Soloway, S., Waltcher, I. \& Leong, B. Competing reactions of electronically excited 1,3-trimethylene dicinnamate. J. Am. Chem. Soc. 94, 7242-7244 (1972). 